\title{
Efeitos do local de deposição do sêmen e do intervalo inseminaçãol ovulação sobre a fertilidade de éguas inseminadas com sêmen fresco diluído
}

\author{
Ivis Luiz Gomes de Sena Xavier ${ }^{1}$, José Monteiro da Silva Filho², Giovanni Ribeiro de \\ Carvalho ${ }^{3}$, Maristela Silveira Palhares ${ }^{2}$, Raissa Rossi ${ }^{1}$, Amanda Pimenta Siqueira ${ }^{4}$
}

\author{
${ }^{1}$ Mestrando em Reprodução Animal, Escola de Veterinária - UFMG. \\ 2 Departamento de Clínica e Cirurgia Veterinárias, Caixa Postal 567-Escola de Veterinária da UFMG, CEP: 30161-970, Belo Horizonte, MG. \\ ${ }^{3}$ Departamento de Zootecnia - UFV. \\ ${ }^{4}$ Doutoranda em Reprodução Animal, Escola de Veterinária - UFMG.
}

RESUMO - Estudou-se o efeito do intervalo da inseminação artificial à ovulação sobre a fertilidade de éguas, considerando principalmente o local de deposição do sêmen. Os intervalos foram de 48 horas e 24 horas antes da ovulação e de 48 horas e 72 horas entre as duas últimas inseminações. As inseminações foram realizadas no ápice do corno uterino, por via intravaginal profunda, com $1 / 5$ da dose inseminante utilizada para o corpo do útero (15 mL-GI). As éguas foram rufiadas diariamente e inseminadas às segundas, quartas e sextas-feiras, a partir de um folículo de 3,0 a 3,5 cm de diâmetro, com sêmen fresco diluído em diluidor de leite desnatado-glicose. Não houve diferença nas taxas de concepção/ciclo entre as inseminações realizadas no corpo (42,86\%) e no ápice (45,95\%) com concentrações médias de 489 e 102 milhões de espermatozoides móveis. Não foram observadas diferenças entre os grupos experimentais no mesmo intervalo inseminação artificial/ovulação, no entanto, quando as taxas de concepção foram comparadas entre intervalos em mesmo local de deposição do sêmen, taxas de concepção inferiores estiveram relacionadas às inseminações realizadas antes da ovulação. Por outro lado, as inseminações realizadas antes e após a ovulação, independentemente dos intervalos inseminação/ovulação, resultaram em melhores taxas de concepção. As concentrações espermáticas/dose inseminante podem ser significativamente reduzidas, sem prejuízo à fertilidade, quando o sêmen é depositado próximo à papila tubárica. Entretanto, há necessidade de inseminações antes e depois da ovulação para que se obtenham melhores taxas de fertilidade quando se utiliza sêmen de baixa viabilidade no trato genital da égua, visando ao estabelecimento de um reservatório espermático adequado no momento da ovulação.

Palavras-chave: ápice do corno uterino, inseminação artificial, sêmen fresco, taxa de concepção

\section{Effects of semen deposition site and the insemination/ovulation interval on fertility of mares inseminated with fresh diluted semen}

\begin{abstract}
The effect of the interval from artificial insemination to ovulation on mare fertility rates was studied, taking account the semen deposition site. The intervals were 48 hours and 24 hours prior to ovulation, 48 hours and 72 hours between the two last inseminations. The inseminations in the tip of the uterine horn (GII) were carried out by the deep intravaginal technique using $1 / 5$ of the inseminating dose used in the uterine body (15mL-GI). The mares were teased daily and inseminated on Mondays, Wednesdays and Fridays, starting with a follicle from 3.0 to $3.5 \mathrm{~cm}$ of diameter, using fresh semen diluted in skim milk glucose extender. Only one stallion of 20 year-old and well-known fertility was utilized. There was no difference in the conception rates/cycles between the inseminations carried out in the uterine body (42.86\%) and in the tip of the uterine horn (45.95\%) utilizing average concentrations of 489 and 102 million mobile spermatozoa, respectively. No differences were observed between experimental groups for the same artificial/ovulation interval, but when the conception rates were between intervals in the same semen deposition place, lower conception rates were associated to inseminations carried out before ovulation. However, artificial insemination carried out before and after ovulation, regardless of the artificial insemination/ovulation intervals, resulted in better conception rates. The spermatic concentrations/inseminating dose can be significantly reduced, without damaging fertility, when the semen is placed close to the tubaric papille. However, inseminations are needed before and after ovulation to obtain better fertility rates when using low viability semen in the mare genital tract, in order to establish an adequate sperm reservoir at the time of ovulation.
\end{abstract}

Key Words: artificial insemination, conception rate, fresh semen, tip of the uterine horn

Recebido em 9/6/2008 e aprovado em 17/3/2009.

Correspondências devem ser enviadas para: silvafilhojm@gmail.com 


\section{Introdução}

A inseminação artificial no equino utilizando baixa concentração espermática/dose inseminante, associada à mudança do local de deposição do sêmen, tem permitido redução drástica do número de células espermáticas necessárias à obtenção de prenhez, por meio da deposição dos espermatozoides próximo à junção útero-tubárica. Acredita-se que a mudança do local de deposição do sêmen tenha potencial para melhorar a eficiência do sêmen congelado ou sexado de certos garanhões (Buchanan et al., 2000; Lindsey et al., 2002a; Lindsey et al., 2002b; Lyle \& Ferrer, 2005). Deve-se considerar ainda o aumento potencial da fertilidade de garanhões subférteis (Rigby et al., 2000) e a utilização do sêmen de garanhões idosos com limitada produção espermática diária, que não permitem a inseminação de todas as éguas em cio durante o pico da estação reprodutiva (Weems \& Byers, 2004).

O efeito do intervalo da inseminação à ovulação é de extrema importância quando se estuda fertilidade (Silva Filho et al., 1998). Quando a inseminação é realizada na fase pré-ovulatória, a fertilização depende essencialmente da viabilidade espermática no trato genital da égua, enquanto na inseminação artificial pós-ovulação dependerá da viabilidade do óvulo. O intervalo de inseminações ou coberturas no manejo reprodutivo pode ser realizado de acordo com a proposta de Silva Filho et al. (1993). Dessa forma, as inseminações artificiais são fixadas às segundas, quartas e sextas-feiras, o que possibilita redução do trabalho nos finais de semana, facilita a programação do técnico no haras e melhora o controle dos dias de inseminação. A aplicabilidade dessa proposta apoia-se em informações de Palhares (1997), Silva Filho et al. (1998) e Saturnino et al. (2002), que sugeriram viabilidade espermática de até 60 horas no trato genital da égua. Procurou-se neste estudo avaliar os efeitos do local de deposição do sêmen e do intervalo inseminação/ovulação sobre a fertilidade de éguas inseminadas com sêmen fresco diluído.

\section{Material e Métodos}

O estudo foi realizado no município de Belo Horizonte, Minas Gerais, localizado a latitude sul 1955", longitude WGR 4356", a uma altitude de 850 metros, com clima do tipo CWA (inverno seco e verão chuvoso), durante os meses de outubro a março.

Foram utilizados 72 ciclos estrais de 37 éguas mestiças, com 4 a 20 anos de idade, pertencentes a diferentes ordens de parto e duas categorias reprodutivas (éguas solteiras e potras). As éguas de faixa etária semelhante foram agrupadas e, em seguida, distribuídas ao acaso, por meio de sorteio, em dois grupos experimentais. A faixa etária das éguas inseminadas no corpo ou no ápice do corno foram de $11,09 \pm 5,07$ e de 11,62 \pm 4,76 anos, respectivamente, utilizando-se 35 e 37 ciclos, na mesma ordem anterior. A inseminação convencional foi realizada no corpo do útero com volume de 15 mL de sêmen. Já a inseminação intracornual profunda foi realizada no ápice do corno uterino, ipsilateral ao folículo dominante, utilizando-se um volume de $3 \mathrm{~mL}$ de sêmen, por intermédio de pipeta flexível guiada via retal, correspondendo a 1/5 da dose inseminante convencional. Todas as éguas foram inseminadas em dias fixos (segundas, quartas e sextas-feiras), de acordo com manejo reprodutivo proposto por Silva Filho et al. (1993), utilizando-se sêmen a fresco diluído de apenas um garanhão.

Durante a análise dos dados, o efeito do intervalo da inseminação artificial à ovulação sobre a fertilidade, de acordo com o local de deposição do sêmen, também foi estudado e os intervalos caracterizados e agrupados da seguinte forma: inseminação artificial 48 horas antes da ovulação em 18 ciclos (todos os ciclos em que a ovulação foi detectada aos domingos); inseminação artificial 24 horas antes da ovulação, em 31 ciclos (todos os ciclos em que a ovulação foi detectada às terças, quintas e sábados); uma inseminação artificial 48 horas antes da ovulação e outra até 24 horas após a ovulação (todos os ciclos em que a ovulação foi detectada às quartas e sextas-feiras, $n=16$ ciclos), ou seja, com intervalo de 48 horas entre as duas últimas inseminações; uma inseminação artificial 72 horas antes da ovulação e outra até 24 horas pós-ovulação, compreendendo todos os ciclos em que a ovulação foi detectada às segundas-feiras, ou seja, com intervalo de 72 horas ( $n=06$ ciclos) entre as duas últimas inseminações.

Esse agrupamento dos intervalos inseminação artificial/ ovulação, proposto anteriormente, dependeu do dia da ocorrência da ovulação, que foi meramente casual. Assim, por ter sido detectado apenas um ciclo com intervalo de 72 horas pré-ovulação (referentes aos ciclos em que a ovulação foi detectada apenas nas segundas-feiras), esse dado foi desconsiderado apenas para as análises referentes aos intervalos inseminação artificial/ovulação, perfazendo número total de 71 ciclos.

De acordo com o proposto por Valle (1997), as variáveis analisadas podem ser classificadas em dois tipos de parâmetros: de controle, com informações sobre a homogeneidade dos tratamentos, como idade das éguas, número de ciclos, número de ciclos por égua, número de inseminação artificial por ciclo, volume de sêmen, volume de diluidor e tempo da colheita/inseminação; e parâmetros de resultados, com informações sobre os resultados 
reprodutivos obtidos a partir dos tratamentos efetuados, incluindo número de inseminações por ciclo positivo; número de inseminações por ciclo negativo; concentração espermática; número de ciclos por concepção; número de ciclos por égua gestante; taxa de concepção por ciclo e taxa de concepção total.

Como doador de sêmen utilizou-se um garanhão da raça Brasileiro de Hipismo com 20 anos de idade e de alto valor genético. No período pré-experimental, após o esgotamento das reservas espermáticas extragonádicas, como recomendado por Pickett et al. (1987), foram realizadas várias colheitas de sêmen, que foi devidamente submetido a avaliações físicas e morfológicas. Apesar das colheitas sequenciadas, observou-se a manutenção de grande número de células mortas no ejaculado, além de rápida queda no vigor e na motilidade espermáticas (aproximadamente 15 minutos), tanto do sêmen in natura como do diluído (1:1), ao serem conservados a $37^{\circ} \mathrm{C}$, em banho-maria. Observou-se também grande sensibilidade das células espermáticas ao resfriamento a $5^{\circ} \mathrm{C}$, por 6 a 12 horas após diluição no diluidor de mínima contaminação, em container proposto por Palhares (1989).

Houve grande diferença entre ejaculados, sobretudo quando os intervalos de colheita ultrapassavam 48 horas. A redução dos intervalos ( $\leq 48$ horas) respondeu por melhor resistência espermática do sêmen diluído e mantido a $37^{\circ} \mathrm{C}$, em banho-maria.

Durante o período pré-experimental a produção de células móveis foi suficiente para inseminar no máximo oito éguas por ejaculado, dentro do preconizado pela literatura como dose inseminante ideal $\left(400 \times 10^{6}\right.$ espermatozoides móveis por dose inseminante), para inseminações no corpo do útero (Pickett et al., 1987). Ainda durante esse período, verificou-se queda sensível da qualidade espermática quando se aumentava o intervalo de ejaculados. Assim, foram propostas colheitas nas segundas, quartas e sextasfeiras, de acordo com sugestões de Palhares (1997), Silva Filho et al. (1998) e Saturnino et al. (2002), além de uma colheita no sábado pela manhã, visando manter a mesma qualidade espermática durante a estação de monta. Estabeleceu-se, nesse momento, a dose a ser utilizada no ápice do corno uterino. Admitiu-se que essa dose fosse suficiente para promover a concepção, em função do quadro espermático apresentado pelo garanhão. Segundo Pace \& Sullivan (1975), Demick et al. (1976), Voss et al. (1979) e Pickett et al. (1987), para inseminações no corpo do útero, o desafio seria em torno de $100 \times 10^{6}$ espermatozoides móveis por dose inseminante. Assim, estabeleceu-se a concentração para o ápice do corno como 1/5 da utilizada no corpo do útero e que, depois de cada ejaculação, o sêmen seria diluído para um total de $120 \mathrm{~mL}$ (diluidor mais sêmen), de forma a compor oito doses de $15 \mathrm{~mL}$. Diante do exposto, a dose inseminante a ser utilizada no ápice do corno uterino, por representar $1 / 5$ da dose utilizada no corpo do útero, foi fixada em $3 \mathrm{~mL}$. Pretendeu-se, também com a redução da dose inseminante para $1 / 5$ da convencional, aumentar o número de doses produzidas por ejaculado, adequando-se as condições do experimento às características seminais de um garanhão senil de alto valor genético, na expectativa de maior eficiência reprodutiva.

Durante a estação de monta, o controle folicular diário, obedeceu ao sistema métrico proposto por Greenhof \& Kenney (1975), com manejo reprodutivo baseado na descrição de Palhares (1987), com adaptações para atender aos objetivos do trabalho. Dessa forma, as éguas foram rufiadas individualmente e palpadas a cada três dias, até 0 início do cio e/ou da presença de um folículo de 2,0-2,5 cm de diâmetro, em um dos ovários; a partir daí, as palpações retais e rufiações tornaram-se diárias até a ovulação. Quando detectada a presença de folículo dominante com diâmetro de 3,0-3,5 cm, as inseminações artificiais foram iniciadas nas segundas, quartas e sextas-feiras até a ovulação.

As inseminações no corpo do útero e ápice do corno uterino foram realizadas por via intravaginal profunda utilizando-se pipeta de inseminação (modelos Provar ${ }^{\circledR}$ e IVI pippette, Minitub ${ }^{\circledR}, 75 \mathrm{~cm}$ ) com o auxílio de palpação retal.

As análises estatísticas foram realizadas por meio da aplicação de modelo que incluiu os efeitos fixos do local de deposição, intervalo inseminação artificial/ovulação e interação dessas variáveis, sobre as variáveis quantitativas.

Os dados proporcionais (número de ciclos/concepção, taxa de concepção/ciclo e taxa de concepção total) foram submetidos ao teste qui-quadrado para detecção de eventuais diferenças entre os tratamentos (Snedecor \& Cochran, 1980).

Comparações das médias da idade das éguas, número de inseminações por ciclo, número de inseminações por ciclo positivo, número de inseminações por ciclo negativo, eficiência de prenhez, volume de sêmen, volume de diluidor, concentração espermática e tempo da colheita à inseminação foram realizadas pelo teste StudentNewman-Keuls (SNK) ou pelo teste t de Student quando se compararam apenas duas médias. Visando obter maior eficiência na detecção de diferenças entre os grupos experimentais, análises de variância foram utilizadas para avaliar os resultados de fertilidade. Por se tratar de uma variável qualitativa, realizou-se a conversão dos dados em quantitativos, segundo proposta de Voss et al. (1975). Por esse método, atribuíram-se pontos a cada ciclo inseminado, da seguinte forma: dez pontos para a concepção no primeiro 
ciclo; oito para concepção no segundo ciclo; seis para o terceiro ciclo; quatro para o quarto ciclo e zero para os ciclos em que não houve concepção. Dessa forma, obteve-se um valor numérico médio para cada grupo experimental, denominado eficiência de prenhez.

\section{Resultados e Discussão}

De acordo com o modelo (Tabela 1), a eficiência de prenhez como variável-dependente foi influenciada $(\mathrm{P}<0,05)$ apenas pelo intervalo inseminação/ovulação. No entanto, a variável não sofreu influência $(\mathrm{P}>0,05)$ do local de deposição do sêmen nem houve interação $(\mathrm{P}>0,05)$ entre local de deposição e intervalo inseminação/ovulação.

Assim, o local de deposição do sêmen não teve efeito $(\mathrm{P}>0,05)$ sobre a fertilidade das éguas, resultando em taxa de concepção/ciclo de 45,95\% (17/37) e 42,86\% (15/35) para inseminações realizadas no ápice do corno uterino e corpo do útero, respectivamente. As taxas de concepção totais obtidas foram de 85\% (17/20) e 83,33\% (15/18), nesta mesma ordem $(\mathrm{P}>0,05)$.

Observou-se claramente como a viabilidade dos gametas pode influenciar as taxas de concepção, o que permite avaliar a exequibilidade das inseminações artificiais em dias fixos da semana, proposta por Silva Filho et al. (1993).

Não foi observada diferença $(P>0,05)$ nas taxas de concepção entre os diferentes locais de deposição do sêmen dentro de um mesmo intervalo inseminação/ovulação (Tabela 2). No entanto, quando as taxas de concepção dos intervalos foram comparadas dentro de mesmo local de

Tabela 1 - Análise de variância da eficiência de prenhez

\begin{tabular}{lc}
\hline Fonte de variação & Valor de P \\
\hline Local de deposição do sêmen & 0,9965 \\
Intervalo inseminação/ovulação (IA/OV) & $0,0013^{*}$ \\
Local de deposição $\times$ inseminação/ovulação & 0,9901 \\
\hline * $<0,05$. &
\end{tabular}

deposição do sêmen, as piores taxas foram encontradas nos intervalos pré-ovulação (24 e 48 horas antes da ovulação).

A taxa de concepção em éguas inseminadas no corpo do útero foi de 33,33\% na inseminação 48 horas antes da ovulação e não diferiu da obtida quando as duas últimas inseminações foram realizadas em intervalo de 48 horas (85,71\%; P=0,053), possivelmente em razão do pequeno número de ciclos avaliados. Esses resultados devem ser interpretados com cautela, porque diferenças reais podem não ter sido detectadas pelo teste de qui-quadrado, em virtude da sobreposição dos intervalos de confiança diante do reduzido número por intervalo inseminação/ovulação. No entanto, o valor da eficiência de prenhez (Tabela 3) confirma a melhor eficiência $(\mathrm{P}<0,05)$ das éguas inseminadas antes e após a ovulação em intervalo de 48 horas $(7,13)$ em comparação à das éguas inseminadas 48 horas préovulação $(2,56)$.

Para a inseminação realizada no ápice do corno uterino, a situação foi semelhante; assim, a análise de qui-quadrado não indicou diferenças $(\mathrm{P}=0,146)$ entre as taxas de concepção dos intervalos de 24 horas antes da ovulação e 72 horas entre as duas últimas inseminações (Tabela 2). Entretanto, a análise de variância (eficiência de prenhez) confirma a melhor eficiência $(\mathrm{P}<0,05)$ do intervalo de 72 horas entre as duas últimas inseminações em relação à inseminação 24 horas antes da ovulação (Tabela 3).

Na presença de pequeno número, observou-se redução na precisão do teste qui-quadrado, em razão da grande sobreposição dos intervalos de confiança. Exemplo disso são as taxas de concepção obtidas com o intervalo de 72 horas entre as duas últimas inseminações em comparação à dos outros intervalos, dentro dos diferentes locais de deposição (Tabela 2). Com isso, diferenças entre os valores obtidos com inseminação realizada 48 horas antes da ovulação (25\%) e 72 horas entre as duas últimas ovulações (75\%) não foram observadas, em razão do baixo número de ciclos avaliados por intervalo.

Tabela 2 - Efeito do intervalo inseminação/ovulação sobre a taxa de concepção de éguas inseminadas no corpo do útero ou ápice do corno uterino com sêmen a fresco diluído

\begin{tabular}{|c|c|c|c|c|}
\hline \multirow[t]{3}{*}{ Intervalo inseminação/ovulação } & \multicolumn{4}{|c|}{ Tratamento } \\
\hline & \multicolumn{2}{|c|}{ Corpo do útero } & \multicolumn{2}{|c|}{ Ápice do corno } \\
\hline & $\mathrm{N}^{\circ}$ de ciclos & Taxa de concepção (\%) & $\mathrm{N}^{\circ}$ de ciclos & Taxa de concepção (\%) \\
\hline 48 horas antes da ovulação & $06(02)$ & $33,33 \mathrm{AB}$ & $12(03)$ & $25,00 \mathrm{~A}$ \\
\hline 24 horas antes da ovulação & $19(04) \mathrm{a}$ & $21,05 \mathrm{~A}$ & $12(04)$ & $33,33 \mathrm{~A}$ \\
\hline 48 horas entre as duas últimas inseminações & $07(06)$ & $85,71 \mathrm{~B}$ & $09(07)$ & $77,78 \mathrm{~B}$ \\
\hline 72 horas entre as duas últimas inseminações & $02(02)$ & $100,00 \mathrm{~B}$ & $04(03)$ & $75,00 \mathrm{AB}$ \\
\hline Total & $34(14)$ & 41,18 & 37 (17) & 45,95 \\
\hline
\end{tabular}

Letras maiúsculas distintas na mesma coluna diferem $(\mathrm{P}<0,05)$ pelo teste qui-quadrado.

a Números entre parênteses referem-se às éguas que ficaram gestantes a cada ciclo. 
Entretanto, conforme os dados (Tabelas 2 e 3), uma dose pós-ovulação é necessária para que o sêmen de baixa viabilidade no trato genital da égua possa resultar em taxas de concepção aceitáveis. Assim, as baixas taxas de fertilidade obtidas com as inseminações realizadas antes da ovulação dentro de 48 a 24 horas (variação de 21,05 a 33,33\%) refletem eficiência de prenhez de 2,19 e 2,56, respectivamente, para as inseminações realizadas nos mesmos intervalos anteriores. Os resultados de fertilidade por ciclo obtidos com inseminações realizadas 24 horas antes da ovulação estão aquém dos $60 \%$ recomendados na literatura e reforçam a baixa viabilidade espermática no sistema genital das éguas. Reforça também a importância de inseminações depois da ovulação quando da utilização de garanhões com sêmen com essas características.

Os resultados de fertilidade obtidos neste estudo são desapontadores em comparação aos apresentados por outros autores (Tabelas 2 e 3). A taxa de concepção obtida para éguas inseminadas 48 pré-ovulação variou de 25 a 33\% e foi inferior àquelas alcançadas por Hammond (1938), Skatkin (1943), Kedrov (1944), Cheng (1964) e Woods et al. (1990), entre 58,8\% e 68,5\%. Para as éguas inseminadas 24 horas pré-ovulação, as taxas entre 21,05 e 33,33\% foram inferiores às encontradas por Saltzman (1940), Skatkin (1943), Kedrov (1944), Cheng (1964), Palmer \& Frauquenot (1984) e Kloppe et al. (1988), de 58,8 a 78\%. Mesmo quando comparados aos resultados obtidos com a utilização de inseminações 72 horas antes da ovulação (Bain, 1957; Palmer \& Frauquenot, 1984; Woods et al., 1990), que variaram de 50 a $60 \%$, os dados obtidos neste estudo ainda foram mais baixos. Alguns autores realizaram inseminações ou cobrições cinco a nove dias préovulação e obtiveram taxas de concepção de 93 e 52\%, respectivamente (Burkhardt, 1949; Bain, 1957). Aliev (1981) inseminou éguas com sêmen congelado em péletes no intervalo de 24 a 13 horas pré-ovulação e obteve $76 \%$ de fertilidade, que diferiu $(\mathrm{P}<0,05)$ dos $20 \%$ de concepção quando as éguas foram inseminadas entre 48 a 37 horas antes da ovulação.

Comparando as taxas de fertilidade entre 72 horas e 48 horas pré-ovulação, Voss et al. (1979), Silva Filho (1994) e Palhares (1997) não observaram diferenças entre esses intervalos. Entretanto, Ferreira (1993) obteve melhores $(\mathrm{P}<0,01)$ taxas de concepção ao primeiro ciclo para éguas inseminadas 24 horas pré-ovulação em relação às inseminadas 48 horas antes da ovulação. Apesar do efeito da diversidade metodológica desses experimentos, além de a sobrevivência espermática ser influenciada pela composição de fluidos luminais (Hunter, 1990), pela individualidade e pelos tipos de tratamento seminal (sêmen a fresco, resfriado ou congelado), as taxas de concepção foram altas, maiores que as obtidas neste estudo (Tabela 2).

Houve semelhança apenas com os estudos conduzidos por Aliev (1981), Katila (1996) e Sieme et al. (2003), que trabalharam com sêmen congelado e obtiveram 12,5 a 20\%, $23 \%$ e 20\%, respectivamente, para as inseminações realizadas 48 horas antes da ovulação. Possivelmente, a menor interação espermatozoide-epitélio uterino foi também uma das causas para a redução da sobrevivência espermática no trato genital da égua quando utilizado sêmen congelado (Scott et al., 2000). No entanto, neste estudo, mesmo utilizando sêmen a fresco diluído, a baixa viabilidade espermática no trato genital feminino resultou em baixas taxas de concepção nos intervalos pré-ovulação. Essa vabilidade espermática é predeterminada pelas características observadas in vitro, representadas pela impossibilidade do resfriamento, pelo grande número de células mortas no ejaculado, pela baixa motilidade, pela rápida queda no vigor espermático após a diluição e pela conservação a $37^{\circ} \mathrm{C}$, um quadro clássico de senilidade reprodutiva do garanhão.

Segundo Hunter (1990), a heterogeneidade das células espermáticas em diferentes estádios de maturação pode compensar a inseminação pré-ovulatória em relação à ovulação. Além disso, a viabilidade espermática está aumentada no trato genital feminino sob domínio estrogênico. No entanto, Silva Filho et al. (1998) consideraram que, nas situações em que a qualidade espermática não permite sua viabilidade por 48-72 horas até a ovulação, a inseminação artificial após ovulação pode suprir a deficiência espermática, fazendo com que a associação de inseminações artificiais antes e após a ovulação proporcione maior segurança no momento da ovulação.

Com base nos resultados encontrados, pode-se dizer que as maiores taxas de concepção foram obtidas a partir

Tabela 3 - Eficiência de prenhez de éguas inseminadas no corpo ou corno uterino com diferentes concentrações espermáticas utilizandose sêmen fresco diluído em diversos intervalos inseminação/ovulação

\begin{tabular}{lccc}
\hline Item & & Intervalo inseminação/ovulação & \\
\cline { 2 - 4 } & $\begin{array}{c}48 \text { antes } \\
\text { da ovulação }\end{array}$ & $\begin{array}{c}24 \text { horas antes } \\
\text { da ovulação }\end{array}$ & $\begin{array}{c}48 \text { horas entre as duas } \\
\text { últimas inseminações }\end{array}$ \\
\hline Eficiência de prenhez & $2,56 \pm 4,33 a c$ & $2,19 \pm 4,04 \mathrm{a}$ & $7,13 \pm 3,79 \mathrm{~b}$ \\
\hline
\end{tabular}

Letras minúsculas distintas na mesma linha diferem $(\mathrm{P}<0,05)$ pelo teste SNK. 
das inseminações realizadas antes e após a ovulação, independentemente dos intervalos inseminação/ovulação avaliados, em comparação às inseminações pré-ovulação (Tabelas 2 e 3), o que confirma a baixa viabilidade espermática no trato genital feminino dos espermatozoides do garanhão utilizado no experimento.

Resultados de fertilidade semelhantes aos deste estudo foram observados por outros autores (Palhares, 1997; Silva Filho et al., 1998; Saturnino et al., 2002) em éguas inseminadas em intervalos de 48 e 72 horas entre as duas últimas inseminações. De acordo com esses autores, as taxas de concepção por ciclo foram de 63,66 e 44,44\%; 55,56 e 55,56\%; 53,45 e 56,76\%, respectivamente, e os intervalos não influenciaram $(\mathrm{P}>0,05)$ a fertilidade das éguas inseminadas. No entanto, esses autores não determinaram se essa taxa de fecundação foi provocada pelos espermatozoides oriundos das inseminações antes ou após a ovulação. Caso a fecundação tivesse ocorrido em função da inseminação realizada antes da ovulação, possivelmente seria resultado da sobrevivência espermática de aproximadamente 36 e 60 horas para os intervalos 48 e 72 horas entre as duas últimas inseminações, respectivamente. Neste estudo, as inseminações após a ovulação parecem ter suprido a deficiência espermática e garantido boas taxas de concepção, em razão da baixa viabilidade espermática no trato genital da égua, indicada pelas baixas taxas de concepção dos intervalos de 24 e 48 horas antes da ovulação (Tabela 2).

Assim, a viabilidade do oócito parece ter garantido boas taxas de fertilidade nas inseminações realizadas após a ovulação. As taxas de concepção obtidas neste experimento são semelhantes aos resultados dos trabalhos de Saltzman (1940), Cheng (1964), Martin et al. (1979), Allen (1981), Belling (1984), Koskinen (1990), Woods et al. (1990), Ferreira (1993) e Jacob et al. (2000) com taxas de 63 a 100\%, respectivamente, quando avaliaram a fertilidade de cobrições pós-ovulação. Entretanto, Palmer \& Frauquenot (1984), Koskinen et al. (1990) e Woods et al.(1990) observaram menores taxas de concepção para inseminações realizadas a partir de 12 horas após a ovulação.
Na tentativa de reduzir o intervalo da inseminação de reforço à ovulação, mesmo sob controle folicular realizado a cada 24 horas, as éguas só foram novamente inseminadas quando o ovário apresentava mudanças indicativas de ovulação recente, caracterizada pela natureza friável do ovário ou pelo não-preenchimento da cavidade folicular pós-ovulação, características observadas antes da formação do corpo hemorrágico. É possível que a proximidade entre a inseminação e a ovulação tenha garantido fecundação adequada, o que está de acordo com Woods et al. (1990), que sugeriram que as inseminações relativamente mais próximas da ovulação são mais efetivas. No mesmo sentido, Skatkin (1943) relatou não haver razão para a redução das taxas de concepção de éguas inseminadas dentro de curto período de tempo após a ovulação quando observou fertilidade de $72 \%$ para éguas inseminadas entre 6 e 14 horas após a ovulação.

Uma inseminação de reforço nas éguas inseminadas 24 horas antes da ovulação deve ser feita quando a qualidade espermática não permite sua viabilidade até 24 horas (Skatkin, 1943). Isso pode resultar em melhores taxas de concepção pela garantia de reservatório espermático povoado por espermatozoides em condições fecundantes no momento da ovulação. Nesses casos, não é possível fixar os dias de inseminação, como proposto por Silva Filho et al. (1993). Com isso, a determinação do intervalo ideal entre cobrições exige testes in vitro e in vivo para cada garanhão antes de se implantar qualquer programa de inseminação artificial, principalmente quando se pretende utilizar longos intervalos das inseminações (Palhares, 1997).

Quando se avaliaram os parâmetros de controle (Tabela 4), como idade das éguas, não foram encontradas diferenças $(\mathrm{P}>0,05)$ entre os grupos experimentais. Entretanto, para o número de inseminações por ciclo, de inseminações ciclo positivo, de inseminações por ciclo negativo, as diferenças $(\mathrm{P}<0,05)$ ocorreram meramente ao acaso, uma vez que não é possível predeterminar o dia da ovulação. A significância no tempo da colheita à inseminação merece comentário similar, ou seja, são observações casuais.

Tabela 4 - Parâmetros de controle de éguas inseminadas no corpo ou corno uterino com sêmen fresco diluído e diversas concentrações espermáticas

\begin{tabular}{|c|c|c|c|c|}
\hline \multirow[t]{2}{*}{ Item } & \multicolumn{4}{|c|}{ Intervalo inseminação/ovulação } \\
\hline & $\begin{array}{c}48 \text { antes } \\
\text { da ovulação }\end{array}$ & $\begin{array}{l}24 \text { horas antes } \\
\text { da ovulação }\end{array}$ & $\begin{array}{l}48 \text { horas entre as duas } \\
\text { últimas inseminações }\end{array}$ & $\begin{array}{l}72 \text { horas entre as duas } \\
\text { últimas inseminações }\end{array}$ \\
\hline Inseminações por ciclo & $1,89 \pm 0,83 a$ & $2,06 \pm 0,89 a$ & $3,31 \pm 1,49 b$ & $2,67 \pm 1,21 \mathrm{ab}$ \\
\hline Inseminações por ciclo positivo & $2,00 \pm 0,71 \mathrm{ab}$ & $2,00 \pm 0,76 a$ & $3,23 \pm 1,42 b c$ & $2,80 \pm 1,30$ ac \\
\hline Inseminações por ciclo negativo & $1,85 \pm 0,90 \mathrm{ac}$ & $2,09 \pm 0,95 a$ & $3,67 \pm 2,08 \mathrm{bd}$ & $2,00 \pm 0,00 \mathrm{acd}$ \\
\hline
\end{tabular}

Letras minúsculas distintas na mesma linha diferem $(\mathrm{P}<0,05)$ pelo teste $\mathrm{SNK}$ 


\section{Conclusões}

O local de deposição do sêmen não tem efeito sobre a fertilidade, mas a associação de inseminações antes e após a ovulação é necessária para obtenção de melhores taxas de fertilidade na presença de sêmen de baixa viabilidade no sistema genital de éguas.

\section{Referências}

ALLEN, W.E. Fertility in pony mares after post ovulation service. Equine Veterinary Journal, v.13, n.2, p.134-135, 1981.

ALIEV, A.I. The effect of ovaritropin on reproductive function of mares, and the optimum time of insemination with frozenthawed semen. Animal Breeding Abstracts, v.49, n.12, p.805, 1981.

BAIN, A.M. Estrus and infertility of the thoroughbred mare in Australasian. Journal of the American Veterinary Medical Association, v.131, n.1, p.179-185, 1957.

BELLING JR., T.H. Postovulation breeding and related reproductive phenomena in the mare. Equine Practice, v.6, n.6, p.12-19, 1984.

BUCHANAN B.R.; SEIDEL, JR., G.E.; McCUE, P.M. et al. Insemination of mares with low numbers of either unsexed or sexed spermatozoa. Theriogenology, v.53, n.6, p.1333-1344, 2000.

BURKHARDT, J. Sperm survival in the tract of the mare. Journal of Agricultural Science, v.39, p.201-203, 1949.

CHENG, P.L. The application of some investigations of reprodudtive physiology in horse breeding practice in China. Animal Breeding Abstracts, v.33, n.1, p.58, 1964.

DEMICK, D.S.; VOSS, J.L.; PICKET, B.W. Effect of cooling, storage, glyceralization and spermatozoa numbers on equine fertility. Journal of Animal Science, v.43, n.3, p.633-637, 1976.

FERREIRA, M.F.L. Efeito de diluentes e taxa de resfriamento sobre a motilidade espermática e fertilidade do sêmen de jumentos (Equus asinus). 1993. 67f. Dissertação (Mestrado em Medicina Veterinária) - Escola de Veterinária/Universidade Federal de Minas Gerais, Belo Horizonte.

GREENHOFF, G.H.; KENNEY, R.M. Evalution of reproductive status of nonpregnant mares. Journal of the American Veterinary Medical Association, v.167, n.6, p.449-458, 1975.

HAMMOND, J. Recent scientific research on horse breeding problems. Yorkshire Agricultural Society Journal, v.95, n.11, p.2-16, 1938.

HUNTER, R.H.F. Gamete lifespans in the mare's genital tract. Equine Veterinary Journal, v.22, p.378-379, 1990.

JACOB, J.C.F.; ALBUQUERQUE, F.T.; DOMINGUES, M.B. et al. Taxa de gestação em éguas Mangalarga Marchador inseminadas pós-ovulação. Revista Brasileira de Reprodução Animal, v.24, n.1, p.51-55, 2000.

KATILA, T. Uterine defence mechanism in the mare. Animal Reproduction Science, v.42, n.3, p.197-204, 1996.

KEDROV, V.K. Mating of mare with prolonged heat. Animal Breeding Abstracts, v.13, n.3, p.131, 1944.

KLOPPE, L.H.; VARNER, D.D.; ELMORE, R.C. et al. Effect on the fertilizing capacity of frozen/thawed equine spermatozoa. Theriogenology, v.29, n.2, p.429-439, 1988.

KOSKINEN, E.; LINDEBERG, H.; KUNTSI, H. et al. Fertility of mares after postovulatory insemination. Journal Veterinary Medical Series A, v.37, n.1, p.77-80, 1990.

LINDSEY, A.C.; SCHENK, J.L.; GRAHAN, J.K. et al. Hysteroscopic insemination of low numbers of flow sorted fresh and frozen/ thawed stallion spermatozoa. Equine Veterinary Journal, v.34, n.2, p.121-127, 2002a.
LINDSEY, A.C.; MORRIS, L.H.A.; ALLEN, W.R. et al. Hysteroscopic insemination of mares with low numbers of nonsorted flow sorted spermatozoa. Equine Veterinary Journal, v.34, p.128-132, 2002b.

LYLE, S.K.; FERRER, M.S. Low-dose insemination - Why, when and how. Theriogenology, v.64, n.3, p.572-579, 2005.

MARTIN, J.C.; KLUG, E.; GÜNZEL, A.R. Centrifugation of stallion semen and its storage in large volume straws. Journal Reproduction and Fertility, Supplement 27, p.447-451, 1979.

PACE, M.M.; SULLIVAN, J.J. Effect of timing of insemination, numbers of spermatozoa and extender components on the pregnancy rate in mares inseminated with frozen stallion semen. Journal Reproduction and Fertility, Supplement 23, p.115121, 1975.

PALHARES, M.S. Influência de um novo container para o transporte do sêmen eqüino diluído e resfriado: I Características termodinâmicas e funcionais, IIDesempenho reprodutivo de éguas inseminadas. 1997. 245f. Tese (Doutorado em Ciência Animal) - Escola de Veterinária, Universidade Federal de Minas Gerais, Belo Horizonte.

PALHARES, M.S. Avaliação da atividade ovariana e eficiência reprodutiva de potras e éguas da raça Mangalarga Marchador. 1989. 89f. Dissertação (Mestrado em Medicina Veterinária) - Escola de Veterinária/Universidade Federal de Minas Gerais, Belo Horizonte.

PALHARES, M.S. Fertilidade e controle. Cavalo Machador, v.1, p.9-10, 1987 .

PALMER, E.; FRAUQUENOT. Mesureet prediction de la fertiliité des étalons. Estude méthodologique. In: R. Jarrige e W. MartinRosset . Le Cheval, Reproduction, selection, limentation, exploitation. Paris: INRA, 1984. p.113-127.

PICKETT, B.W.; SQUIRES, E.L.; McKINNON, A.O. Procedures of collections, evaluation and utilization of stallion semen for artificial insemination. Fort Collins: Colorado Stade University, Animal Reproduction Laboratory, 1987. 125p.

RIGBY, S.; DERCZO, S.; BRINSKO, S. et al. Oviductal sperm numbers following proximal uterine horn or uterine body insemination. In: CONVENTION OF THE AMERICAN ASSOCIATION OF EQUINE PRACTITIONERS, 46., 2000, Santo Antônio, Texas. Proceedings... Santo Antônio: AAEP, 2000. p.332-334.

SALTZMAN, A.A. Insemination of mares after ovulation. Animal Breeding Abstracts, v.8, n.1, p.16, 1940.

SATURNINO, H.M.; SILVA FIHO, J.M.; DANTAS, M. et al. Efeito do intervalo entre as duas últimas inseminações sobre a fertilidade de éguas inseminadas com sêmen fresco, diluído. Revista Brasileira de Zootecnia, v.31, p.1143-1149, 2002.

SCOTT, M.A.; LIU, I.K.M.; OVERSTREET, J.W. et al. The structural morphology and epithelial association of spermatozoa at the uterotubal junction: a descriptive study of equine spermatozoa in situ using scanning electro microscopy. Journal of Reproduction and Fertility, Supplement 56, p.415-421, 2000.

SIEME, H.; SCHÄFER, T.; STOUT, T.A.E. et al. The effects of different insemination regimes on fertility in mares. Theriogenology, v.60, n.6, p.1153-1164, 2003.

SILVA FILHO, J.M.; VALLE, G.R.; SATURNINO, H.M. et al. Influência do intervalo entre a inseminação artificial com sêmen diluído resfriado e transportado e a ovulação sobre a fertilidade de éguas. Arquivo Brasileiro de Medicina Veterinária e Zootecnia, v.50, n.5, p.563-568, 1998.

SILVA FILHO, J.M. Avaliação do manejo reprodutivo e do sêmen na inseminação artificial de eqüinos. 1994. 408f. Tese (Doutorado em Zootecnia) - Universidade Federal de Viçosa, Viçosa, MG.

SILVA FILHO, J.M.; SATURNINO, H.M.; PALHARES, M.S. et al. Efeito do intervalo entre as duas últimas inseminações e da concentração espermática sobre a fertilidade de éguas 
inseminadas com sêmen a fresco diluído. In: CONGRESSO BRASILEIRO DE REPRODUÇÃO ANIMAL, 10., 1993, Belo Horizonte. Anais... Belo Horizonte: Colégio Brasileiro de Reprodução Animal, 1993. p.264.

SKATKIN, P.N. Systems of mating and artificial insemination of horses. Animal Breeding Abstract, v.11, n.2, p.86, 1943.

SNEDCOR, G.W.; COCHRAN, W.G. Statistical methods. Ames: Iowa State Universty, 1980. 505p.

VALLE, G.R. Efeito da rufiação e manipulação do sistema genital e transporte de sêmen, em container “celle” modificado, na avaliação da técnica de inseminação artificial de eqüinos. 1997. 349f. Dissertação (Mestrado em Medicina Veterinária) - Escola de Veterinária/Universidade Federal de Minas Gerais, Belo Horizonte.

VOSS, J.L.; WALLACE, R.A.; SQUIRES, E.L. et al. Effects of synchronization and frequency of insemination on fertility.
Journal of Reproduction and Fertility, Supplement 27, p.257-261, 1979.

VOSS, J.L.; PICKETT, B.W.; BACK, D.G. et al. Effect of rectal palpation on pregnancy rate of nonlactating, normally cycling mares. Journal of Animal Science, v.41, n.3, p.829-834, 1975.

WEEMS, J.C.; BYERS, W. How to incorporate low-dose hysteroscopic insemination in a on-farm fresh semen program. In: CONVENTION OF THE AMERICAN ASSOCIATION OF EQUINE PRACTITIONERS, 50., 2004, Lexington. Anais eletrônicos... Lexington: 2004. Disponível em: <http:// www.ivis.org/proccedings/AAEP/2004/weems/chapter.asp?LA=1>. Acesso em: 30/8/2005.

WOODS, J.; BERGFELT, D.R.; GINTHER, O.J. Effects of time of insemination relative to ovulation on pregnancy rate and embryonic-loss rate in mares. Equine Veterinary Journal, v.22, n.6, p.410-415, 1990. 